\title{
QTL analysis of main and epistatic effects for flour color traits in durum wheat
}

\author{
Pablo F. Roncallo • Gerardo L. Cervigni • Carlos Jensen • \\ Rubén Miranda $\cdot$ Alicia D. Carrera $\cdot$ Marcelo Helguera • \\ Viviana Echenique
}

Received: 7 July 2011/Accepted: 20 January 2012/Published online: 7 February 2012

(C) Springer Science+Business Media B.V. 2012

\begin{abstract}
The aim of this work was to map quantitative trait loci (QTLs) associated with flour yellow color $\left(\mathrm{Fb}^{*}\right)$ and yellow pigment content (YPC) in durum wheat (Triticum turgidum L. var. durum). Additionally, QTLs affecting flour redness $\left(\mathrm{Fa}^{*}\right)$ and brightness (FL*) color parameters were investigated. A population of 93 RILs (UC1113 $\times$ Kofa) was evaluated in three locations of Argentina over 2 years. High heritability values ( $>94 \%$ ) were obtained for $\mathrm{Fb}^{*}$ and YPC, whereas $\mathrm{FL}^{*}$ and $\mathrm{Fa}^{*}$ showed intermediate to high values. The main QTLs affecting $\mathrm{Fb}^{*}$ and YPC overlapped on chromosome arms 4AL (4AL.2), 6AL
\end{abstract}

Electronic supplementary material The online version of this article (doi:10.1007/s10681-012-0628-x) contains supplementary material, which is available to authorized users.

P. F. Roncallo · A. D. Carrera · V. Echenique $(\bowtie)$ Laboratorio de Biotecnología Vegetal, CERZOSCONICET/UNS, CCT Bahía Blanca, Camino La Carrindanga km 7, 8000 Bahía Blanca, Argentina e-mail: echeniq@criba.edu.ar

P. F. Roncallo

e-mail: roncallo@criba.edu.ar

P. F. Roncallo · A. D. Carrera - V. Echenique Departamento de Agronomía, Universidad Nacional del Sur, San Andrés 800, 8000 Bahía Blanca, Argentina

G. L. Cervigni CEFOBI-CONICET, Suipacha 531 S2002 LRK, Rosario, Argentina
(6AL.2), 7AS, 7AL, 7BS (7BS.2) and 7BL (7BL.2). The 7BL.1 QTL included the Psy-B1 locus, but one additional linked QTL was detected. A novel minor QTL located on 7AS affected $\mathrm{Fb}^{*}$, with an epistatic effect on YPC. An epistatic interaction occurred between the 7AL and 7BL.2 QTLs. The 4AL.2 QTL showed a strong effect on $\mathrm{Fb}^{*}$ and was involved in two digenic epistatic interactions. The 6AL.2 QTL explained most of the variation for $\mathrm{Fb}^{*}$ and YPC. The main QTLs affecting $\mathrm{FL}^{*}$ and $\mathrm{Fa}^{*}$ were located on $2 \mathrm{BS}$ and $7 \mathrm{BL}$, respectively. These results confirm the complex inheritance of flour color traits and open the possibility of developing perfect markers to improve pasta quality in Argentinean breeding programs.

\author{
C. Jensen \\ INTA.CEI Barrow, Ruta 3 km 487, 7500 Tres Arroyos, \\ Argentina \\ R. Miranda \\ Asociación de Cooperativas Argentinas, Ruta 51 km 710, \\ 8118 Cabildo, Argentina \\ M. Helguera \\ INTA EEA Marcos Juárez, CC 21, 2580 Marcos Juárez, \\ Argentina
}


Keywords Quantitative trait loci (QTLs) - Triticum turgidum L. var. durum - Flour color parameters . Yellow pigment content

\section{Introduction}

Durum wheat (Triticum turgidum L. var. durum) is used in pasta production due to the quality attributes of its grains, principally protein content, gluten strength and yellow color. Yellow pigment content (YPC) mainly caused by the xanthophyll lutein is the most important determinant of the bright yellow color in semolina (Hentschel et al. 2002). However, the final color of pasta is also influenced by the enzymatic degradation of carotenoids by lipoxygenases (Carrera et al. 2007), with at least two additional enzymes (peroxidases and polifenol oxidases) playing secondary roles (Borrelli et al. 2008). Grain debranning and manufacturing conditions are also key factors affecting yellowness (Matsuo and Dexter 1980; Borrelli et al. 2008). Carotenoids have an antioxidant role by reducing oxidative damage to biological membranes. As precursors of vitamin A, carotenoids are valuable nutritional components of pasta products.

Colorimetric determinations of semolina or flour are often used by breeders as tools to select materials with high yellow color. Alternatively, the quantification of carotenoid pigments and lipoxygenase activity in semolina through spectrophotometric determinations can be used. YPC in grains and yellow color of milling products are considered complex heritable traits controlled by several genomic regions (Clarke et al. 2006). The genetic architecture of these traits has been investigated through quantitative trait locus (QTL) analysis in durum and bread wheat using different mapping populations, and a large number of genomic regions were reported on chromosomes 1A (Patil et al. 2008; Zhang et al. 2009), 1B (Zhang et al. 2009), 2A (Pozniak et al. 2007; Blanco et al. 2011), 3A and 3B (Mares and Campbell 2001; Howitt et al. 2009; Blanco et al. 2011), 4A (Zhang et al. 2008), 5A (Hessler et al. 2002; Blanco et al. 2011), 5B (Patil et al. 2008), 6A (Mares and Campbell 2001; Zhang et al. 2006), 6B (Pozniak et al. 2007), 7A (Mares and Campbell 2001; Patil et al. 2008; He et al. 2008; Singh et al. 2009; Zhang et al. 2009; Blanco et al. 2011) and 7B (Elouafi et al. 2001; Pozniak et al. 2007; Zhang and Dubcovsky 2008). However, epistatic effects (digenic epistasis, QQ) and environmental interactions
(QE or QQE) among QTLs affecting YPC and yellow color have been less frequently investigated. Zhang and Dubcovsky (2008) and Singh et al. (2009) reported analyses of epistatic interactions using two-way ANOVA. Several strategies were proposed to study QTL $\times$ environment interactions (Piepho 2000), or epistatic effects (Kao et al. 1999; Yi et al. 2003) separately, but it is also possible to integrate both effects into the framework of a mixed linear model (Wang et al. 1999; Yang et al. 2007).

Molecular characterization of the carotenoid biosynthetic pathway in wheat has recently been explored. Most of the effort was focused on the Psyl gene, coding for the enzyme phytoene synthase 1 (PSY1), which is considered the rate-limiting step of the pathway (Hirschberg 2001). QTL mapping analysis showed that the Psyl gene co-segregates with YPC and flour yellow color $\left(\mathrm{Fb}^{*}\right)$ on homoeologous chromosomes 7BL (Pozniak et al. 2007; Zhang and Dubcovsky 2008) and 7AL (Howitt et al. 2009; Singh et al. 2009). Allelic variants of $P s y l$ (Psy-Al and $P$ sy-Bl) were reported in durum (Singh et al. 2009; He et al. 2009a; Zhang and Dubcovsky 2008) and common (He et al. 2009b; Howitt et al. 2009; Crawford et al. 2011) wheat.

A second Psy functional gene (Psy2) was located on group 5 chromosomes of durum wheat through genetic and physical mapping, but it could not be associated with YPC (Pozniak et al. 2007; Blanco et al. 2009). In hexaploid wheat, full-length cDNAs for two additional enzymes (phytoene desaturase [PDS] and $\zeta$-carotene desaturase [ZDS]) were cloned (Cong et al. 2009). These enzymes are responsible for four carotene desaturation steps of phytoene in the carotenoid biosynthesis pathway, producing lycopene. Cenci et al. (2004) mapped PDS and ZDS clones from a durum wheat BAC library on chromosome groups 4 and 2, respectively. Recently, Zhang et al. (2011) cloned the full-length DNA sequence of a ZDS gene, located on chromosome 2DL, and a functional marker was developed. Additionally, the lycopene $\varepsilon$-cyclase ( $\varepsilon$-LCY) gene, responsible for cyclization of lycopene, was proposed by Howitt et al. (2009) as a candidate gene for a QTL on chromosome 3B affecting lutein content in common wheat (Mares and Campbell 2001).

In the present work, we focused on validation of QTLs associated with $\mathrm{Fb}^{*}$ and YPC in Argentina, using a durum wheat mapping population derived from the cross UC1113 $\times$ Kofa previously evaluated in the USA, and searched for new and additional QTLs 
expressed in these environments. We used single-locus and two-loci QTL analyses to detect main effects, digenic epistasis (QQ) and QTL $\times$ environment interactions (QE and $\mathrm{QQE})$. Understanding the different genes/QTLs and their interactive effects is essential for determining optimal strategies for marker assisted selection (MAS). We also investigated the presence of QTLs associated with brightness and redness of flour. Additionally, the relationships between color traits and other quality and yield traits were explored. Our final objective was to use this information in Argentinean durum wheat breeding programs to improve flour color.

\section{Materials and methods}

\section{Plant materials}

A mapping population consisting of 93 recombinant inbred lines (RILs) was obtained by crossing the line UC1113 with the variety Kofa (Zhang et al. 2008). UC1113, a breeding line from the UC Davis Wheat Breeding Program, has excellent agronomic performance, but intermediate pasta quality parameters. Kofa, is a durum variety developed by West-Bred, has optimal semolina and pasta color, high protein content and strong gluten. Eight Argentinean durum cultivars were included as controls in all experiments (Buck Platino, Buck Topacio, Buck Esmeralda, Buck Cristal, Buck Ambar, Bonaerense INTA Facon, Bonaerense INTA Carilo and Bonaerense INTA Cumenay).

Field trials

The 93 RILs, along with the parental lines and control varieties, were evaluated over two consecutive years (2006 and 2007) at three locations in Argentina (Cabildo [CA] $\left(39^{\circ} 36^{\prime} \mathrm{S} 61^{\circ} 64^{\prime} \mathrm{W}\right)$, Barrow [BW] $\left(38^{\circ} 20^{\prime} \mathrm{S} 60^{\circ} 13^{\prime} \mathrm{W}\right)$ and Balcarce [BC] $\left(37^{\circ} 45^{\prime} \mathrm{S}\right.$ $\left.58^{\circ} 18^{\prime} \mathrm{W}\right)$ ). The experimental design consisted of randomized complete blocks with three replications using plots $3 \mathrm{~m}^{2}$ in size (3 rows of $5 \mathrm{~m}$ length, $0.20 \mathrm{~m}$ evenly spaced). Seed was sown at an average of 150 per $\mathrm{m}^{2}$. Each year $\times$ location combination was considered as an environment (E). Agronomic management was performed according to local practices at each location. Fertilization was performed in two applications, at pre-sowing/sown and tillering [described in Conti et al. (2011)].
Quality trait evaluations

Approximately 20-25 g of clean grain from each plot was ground to wholemeal flour in a UDY Cyclone Mill (Udy Corporation, Fort Collins, CO, USA) fitted with $1 \mathrm{~mm}$ sieve. Color parameters (CIE L*a*b*) were measured on the wholemeal flour within $48 \mathrm{~h}$ with a Minolta colorimeter (model CR310, Minolta Corp., Ramsey, NJ, USA). CIE L*a*b* tristimulus values were expressed within the tridimensional color space CIE 1976 (Commission Internationale de l'Éclairage, 1976). Positive values of CIE $b *$ represent the yellow color $\left(\mathrm{Fb}^{*}\right)$ of wholemeal flour, while positive values of CIE a* describe the redness of samples ( $\left.\mathrm{Fa}^{*}\right)$. CIE $\mathrm{L}^{*}$ values indicate the brightness of flour (FL*), ranging from 0 (black) to 100 (white).

Wholemeal flour samples were analyzed for YPC using the microtest described by Fares et al. (1991) during the week after milling. Yellow pigments were extracted from $1 \mathrm{~g}$ of wholemeal flour using $5 \mathrm{ml}$ of water-saturated $n$-butanol, by shaking on a rocker table at room temperature for $3 \mathrm{~h}$ at $170 \mathrm{rpm}$. The extracted solution was filtered through Whatman No 1 paper and the absorbance was measured on the supernatant at $448 \mathrm{~nm}$ using a spectrophotometer (METROLAB 1600 PLUS, version 3.06b). YPC was expressed as $\mu \mathrm{g} / \mathrm{g}$ using the formula $\mathrm{c}=[\mathrm{E}$ (extinction at $448 \mathrm{~nm}) \times$ Volume $(\mathrm{ml}) \times 1,000] /[251 \times \mathrm{g}$ (sample weight) $\times \mathrm{s}$ (optical path length)].

Additional quality and yield traits were included in order to examine their relationship with color traits. Thousand kernel weight (TKW) was obtained by weighing two samples of 100 seeds. Test weight (TW) was performed using a hectolitric balance and expressed in $\mathrm{kg} \mathrm{hl}^{-1}$. Grain yield (GY) of each plot was measured as the weight of clean grains from the entire plot area and the results were expressed in $\mathrm{kg} \mathrm{ha}^{-1}$. Grain protein content (GPC) and moisture were measured by the NIRT method using Infratec 1226 equipment (Tecator, Sweden). Gluten strength was estimated on wholemeal flour samples using the SDS sedimentation test (mm) modified by Dick and Quick (1983).

Statistical analysis

Statistical analysis was conducted using the SAS 9.0 software (SAS Institute Inc.; Cary, NC, USA). The normality of the residuals was confirmed by the ShapiroWilk test. Analyses of variance (ANOVA) were 
performed using the PROC MIXED procedure, considering genotype, environments and interactions as random effects. Broad sense heritability $\left(h^{2}\right)$ was estimated using the GENES software (http://www.ufv.br/dbg/genes/ genes.htm). Pearson correlation coefficients between color traits with different yield and quality parameters were calculated using the PROC CORR procedure (Base SAS $^{\circledR}$ 9.2 Procedures Guide, 2010).

QTL mapping

QTL mapping was performed using a genetic map of 269 markers spanning 2,140 cM and including molecular (230 SSR, 23 SNP, 10 RFLP and 3 STS), morphological (Bla) and protein markers (Glu-B1 and Gli-A2 loci) (Zhang et al. 2008). The $X$ letter preceding the marker name that is generally used to indicate the marker type (McIntosh et al. 2003) was omitted from the text and tables. Main effect QTLs were detected for each environment using the Windows QTL-Cartographer software version 2.5 (Wang et al. 2004). Composite Interval Mapping (CIM) was implemented by the standard Model 6, with a $0.5 \mathrm{cM}$ walking speed and a $10 \mathrm{cM}$ window size. A QTL was declared significant when the LOD value was higher than the threshold calculated based on 1,000 permutations at significance level of 0.05 and suggestive at $p<0.1$ (Churchill and Doerge 1994). The confidence intervals were calculated as the two-LOD drop off support intervals that confer a 95\% confidence region (Van Ooijen 1992). To explore the genetic architecture of color traits the QTLNetwork version 2.0 (http://ibi.zju.edu.cn/software/qtlnetwork/) was used. Based on a mixed linear model (Wang et al. 1999), single-locus and two-loci QTL analyses were performed to examine main effects, epistatic effects (QQ) and QTL $\times$ environment interactions (QE and QQE). A genome scan configuration with a walk speed of $0.5 \mathrm{cM}$ and a window size of $10 \mathrm{cM}$ was selected. The critical threshold value of the $F$-statistic was determined by the 1,000 permutation test at a significance level of 0.05 .

\section{Results}

Analysis of phenotypic data

The values of color parameters CIE L*a*b* and YPC showed transgressive bi-directional segregation in the six environments and in the pooled data (Table 1).
Considering the average values of all six environments, the RILs ranged from 4.17 to $7.93 \mathrm{ppm}$ for YPC and 14.27 to 17.71 color units for $\mathrm{Fb}^{*}$. Normal distributions of values for each trait were confirmed by ShapiroWilk tests in four environments. Kofa showed higher $\mathrm{Fb}^{*}$ and YPC values than UC1113 in all environments. In most of them, values for Kofa were slightly lower than the corresponding values for Buck Topacio, the best local variety regarding this trait. In general, Buck Topacio, followed by BI Facon and BI Carilo had the highest $\mathrm{Fb}^{*}$ and YPC (Tables 1 and S1). However, two promising RILs (numbers 32 and 42) performed better than Buck Topacio in most of the environments.

The combined ANOVA showed that genotype and environment effects were highly significant $(p<$ 0.0001 ) for all color traits (Table S2). This analysis also revealed that genotype $\times$ environment interactions were highly significant for all traits. However, there were moderate to high correlations among environments (ranging from 0.61 to 0.78 for $\mathrm{Fb}^{*}$ and 0.70 to 0.89 for YPC). The lowest $\mathrm{Fb}^{*}$ and YPC values were observed in Balcarce (Table 1). Intermediate to high values were found in Barrow, whereas Cabildo showed the highest $\mathrm{Fb}^{*}$ and YPC values (Table 1). High broad sense heritabilities $(\geq 94 \%)$ for YPC and $\mathrm{Fb}^{*}$ were obtained in all the environments, whereas FL* (brightness) and $\mathrm{Fa}^{*}$ (redness) showed intermediate to high heritability values (Table 1 ).

The associations among color traits and with other quality and yield parameters (Table S3) were also investigated. $\mathrm{Fb}^{*}$ and YPC were highly and positively correlated $(r=0.83, p<0.0001)$. Both parameters, as well as $\mathrm{Fa}^{*}$, were highly significantly $(p<0.0001)$ and negatively correlated with GY (Fa*, $r=-0.32$; $\mathrm{Fb}^{*}, r=-0.11$; YPC, $\left.r=-0.27\right)$, TKW (Fa*, $\left.r=-0.35 ; \mathrm{Fb}^{*}, r=-0.65 ; \mathrm{YPC}, r=-0.55\right)$ and TW (Fa*, $r=-0.58 ; \mathrm{Fb}^{*}, r=-0.49$; YPC, $r=$ $-0.51)$. Correlations between protein content (GPC) and color traits (YPC and $\mathrm{Fb}^{*}$ ) were highly significant, with moderately positive values $(r=0.41$ and 0.51 , $p<0.0001)$. There was an unexpected highly significant $(p<0.0001)$ negative correlation between red color $\left(\mathrm{Fa}^{*}\right)$ and SDS test values $(r=-0.41)$.

Identification of QTLs controlling $\mathrm{Fb}^{*}$ and YPC

QTL mapping of the UC1113 × Kofa RIL population showed a high number of chromosomal regions involved in the expression of the correlated traits. 
Table 1 Phenotypic data analysis and heritability values $\left(h^{2}\right)$ of color parameters and YPC for the UC1113 $\times$ Kofa RIL population and parental lines across six environments in Argentina

\begin{tabular}{|c|c|c|c|c|c|c|c|c|}
\hline Trait $^{\mathrm{a}}$ & Environment $^{\mathrm{b}}$ & RILs min. & RILs mean ${ }^{c}$ & RIL máx. & $\mathrm{SD}^{\mathrm{d}}$ & Kofa & UC1113 & Heritability $\left(h^{2}\right)$ \\
\hline \multirow[t]{7}{*}{ FL* } & CA 2006 & 79.06 & $81.38 \mathbf{c}$ & 83.41 & 0.67 & 81.86 & 82.18 & 0.75 \\
\hline & BW 2006 & 78.28 & $80.49 \mathbf{e}$ & 82.47 & 0.67 & 81.85 & 82.17 & 0.75 \\
\hline & BC 2006 & 78.13 & $80.87 \mathbf{d}$ & 83.02 & 0.78 & 80.71 & 81.55 & 0.42 \\
\hline & CA 2007 & 80.21 & $82.33 \mathbf{b}$ & 84.77 & 0.89 & 83.01 & 82.84 & 0.88 \\
\hline & BW 2007 & 79.33 & $81.39 \mathbf{c}$ & 82.85 & 0.59 & 81.40 & 82.33 & 0.69 \\
\hline & BC 2007 & 80.38 & $82.98 \mathbf{a}$ & 85.21 & 0.88 & 83.45 & 83.23 & 0.89 \\
\hline & Mean & 80.53 & 81.57 & 82.33 & 0.39 & 82.04 & 82.38 & 0.68 \\
\hline \multirow[t]{7}{*}{$\mathrm{Fa}^{*}$} & CA 2006 & -0.18 & $0.62 \mathbf{a}$ & 1.50 & 0.28 & -0.18 & 0.11 & 0.87 \\
\hline & BW 2006 & -0.46 & $0.46 \mathbf{b}$ & 1.38 & 0.40 & -0.16 & 0.19 & 0.94 \\
\hline & BC 2006 & -0.63 & $0.31 \mathrm{c}$ & 1.47 & 0.31 & -0.25 & -0.04 & 0.69 \\
\hline & CA 2007 & -0.90 & $-0.06 \mathbf{d}$ & 1.01 & 0.36 & -0.70 & -0.16 & 0.90 \\
\hline & BW 2007 & -0.63 & $-0.04 d$ & 0.83 & 0.23 & -0.06 & 0.01 & 0.73 \\
\hline & BC 2007 & -0.89 & $-0.18 \mathbf{e}$ & 0.53 & 0.29 & -0.44 & 0.02 & 0.90 \\
\hline & Mean & -0.20 & 0.18 & 0.57 & 0.17 & -0.30 & 0.02 & 0.63 \\
\hline \multirow[t]{7}{*}{$\mathrm{Fb}^{*}$} & CA 2006 & 15.00 & $17.26 \mathbf{a}$ & 19.54 & 0.82 & 18.13 & 16.76 & 0.95 \\
\hline & BW 2006 & 12.31 & $16.04 d$ & 18.50 & 0.93 & 16.78 & 15.17 & 0.96 \\
\hline & BC 2006 & 13.71 & $15.57 \mathrm{e}$ & 17.66 & 0.78 & 16.70 & 15.11 & 0.95 \\
\hline & CA 2007 & 13.68 & $16.44 c$ & 18.50 & 0.83 & 16.91 & 15.95 & 0.95 \\
\hline & BW 2007 & 14.65 & $16.55 b$ & 18.71 & 0.87 & 17.56 & 15.62 & 0.94 \\
\hline & BC 2007 & 12.18 & $14.38 \mathbf{f}$ & 16.24 & 0.84 & 15.45 & 13.65 & 0.96 \\
\hline & Mean & 14.27 & 16.03 & 17.73 & 0.73 & 16.92 & 15.38 & 0.91 \\
\hline \multirow[t]{7}{*}{ YPC } & CA 2006 & 4.74 & $6.67 \mathbf{b}$ & 9.36 & 0.92 & 7.56 & 5.60 & 0.97 \\
\hline & BW 2006 & 4.34 & $6.75 \mathbf{a}$ & 9.66 & 0.98 & 7.66 & 5.66 & 0.97 \\
\hline & BC 2006 & 3.23 & $5.56 \mathrm{e}$ & 7.43 & 0.86 & 6.16 & 4.45 & 0.98 \\
\hline & CA 2007 & 4.14 & $6.23 \mathrm{c}$ & 8.43 & 0.84 & 7.03 & 5.57 & 0.98 \\
\hline & BW 2007 & 3.94 & $5.94 d$ & 8.13 & 0.86 & 7.02 & 5.08 & 0.97 \\
\hline & BC 2007 & 3.33 & $4.81 \mathbf{f}$ & 6.67 & 0.69 & 5.68 & 3.86 & 0.98 \\
\hline & Mean & 4.17 & 5.99 & 7.93 & 0.78 & 6.85 & 5.04 & 0.97 \\
\hline
\end{tabular}

${ }^{a} F L^{*}$ flour brightness, $F a^{*}$ flour redness, $F b^{*}$ flour yellow color, $Y P C$ yellow pigment content $\left(\mu \mathrm{g} \mathrm{g}^{-1}\right)$

b $C A$ Cabildo, $B W$ Barrow, $B C$ Balcarce, Mean pooled data of the six environments

${ }^{c}$ Mean values of RILs with the same letter are not significantly different according to the Duncan test at $p<0.01$ (for each trait)

d $S D$ standard deviation

Sixteen QTLs were detected across environments, and major overlapping QTLs (explaining more than $10 \%$ of the phenotypic variation) affecting $\mathrm{Fb}^{*}$ and $\mathrm{YPC}$ were located on chromosome arms 4AL, 6AL, 7AS, 7AL, 7BS and 7BL (Tables 2, 3). However, only 2-5 QTLs per environment/trait combination were detected.

\section{Chromosome arm 1BL}

One QTL was detected on 1BL with positive alleles provided by Kofa. The QYpc.cerz-1BL.1 peak was mapped between BE443797_436 and barc302, and the QTL explained $10.2 \%$ of the phenotypic variation for
YPC in BW 2006 (Table 3). Mapping analysis using the average of the six environments also detected a suggestive $(p<0.1)$ QTL in this region associated with $\mathrm{Fb}^{*}$ (QFb.cerz-1BL.1), but failed to detect an effect in this region associated with YPC (Tables 2, 3; Fig. S1).

\section{Chromosome arm $4 A L$}

Two different QTLs were detected on AAL, with one positive allele provided by UC1113 (4AL.1) and one by Kofa (4AL.2). QYpc.cerz-4AL. 1 lying between $L p x$ $A 3$ and $w m c 617$ was associated with YPC in one environment (CA 2006) and with $\mathrm{Fb}^{*}$ in two environments 
Table 2 QTL mapping of flour yellow color $\left(\mathrm{Fb}^{*}\right)$ in a RIL population (UC1113 $\times$ Kofa) in six environments in Argentina

\begin{tabular}{|c|c|c|c|c|c|c|c|c|}
\hline $\begin{array}{l}\text { Chromosome } \\
\text { arm }\end{array}$ & QTL $^{\mathrm{a}}$ & Flanking markers ${ }^{\mathrm{b}}$ & $\mathrm{LOD}^{\mathrm{c}}$ & $\begin{array}{l}\text { Additive } \\
\text { effect }^{\mathrm{d}}\end{array}$ & $\begin{array}{l}R^{2} \\
(\%)^{\mathrm{e}}\end{array}$ & $\begin{array}{l}\text { Peak } \\
\text { position } \\
(\mathrm{cM})\end{array}$ & $\begin{array}{l}\text { 2-LOD } \\
\text { support } \\
\text { interval }\end{array}$ & Environment $^{\mathrm{f}}$ \\
\hline $1 \mathrm{BL}$ & QFb.cerz-1BL.1 & BE443797_436-barc302 & $2.8 \mathrm{~s}$ & 0.22 & 8.6 & 51.6 & $34.9-60.5$ & Mean \\
\hline $4 \mathrm{AL}$ & QFb.cerz-4AL.1 & dupw4-barc170 & $3.4^{*}$ & -0.24 & 9.1 & 45.7 & $31.6-57.8$ & CA 2006 \\
\hline $4 \mathrm{AL}$ & QFb.cerz-4AL.1 & dupw4-barc170 & $3.6^{*}$ & -0.26 & 8.4 & 46.2 & $5.3-49.7$ & BW 2006 \\
\hline $4 \mathrm{AL}$ & QFb.cerz-4AL.2 & wmc219-psr573.2 & $3.2^{*}$ & 0.23 & 8.3 & 126.2 & $109.4-129.6$ & CA 2006 \\
\hline $4 \mathrm{AL}$ & QFb.cerz-4AL.2 & wmc219-pr573.2 & $3.5^{*}$ & 0.26 & 10.4 & 126.2 & $114.4-129.6$ & CA 2007 \\
\hline $4 \mathrm{AL}$ & QFb.cerz-4AL.2 & wmc219-psr573.2 & $3.0^{*}$ & 0.22 & 6.7 & 126.2 & $113.9-129.6$ & BW 2007 \\
\hline $4 \mathrm{AL}$ & QFb.cerz-4AL.2 & wmc219-psr573.2 & $4.0^{*}$ & 0.24 & 10.6 & 126.2 & $113.9-129.6$ & Mean \\
\hline $5 \mathrm{AS}$ & QFb.cerz-5AS & wmc350-gwm47 & $4.5^{*}$ & -0.3 & 12.9 & 2.0 & $0.0-16.0$ & BW 2007 \\
\hline $5 \mathrm{AS}$ & QFb.cerz-5AS & wmc350-gwm47 & $5.1^{*}$ & -0.34 & 16.2 & 0.0 & $0.0-8.0$ & BC 2007 \\
\hline $5 \mathrm{BL}$ & QFb.cerz-5BL.1 & barc74-gwm371 & $4.3^{*}$ & -0.29 & 12.2 & 42.7 & $23.1-53.2$ & BW 2007 \\
\hline $6 \mathrm{AL}$ & QFb.cerz-6AL.1 & barc146-gwm132 & $5.7 *$ & 0.37 & 16.1 & 36.2 & $23.3-47.4$ & BW 2006 \\
\hline $6 \mathrm{AL}$ & QFb.cerz-6AL.1 & barc146-gwm132 & $5.8^{*}$ & 0.35 & 18.4 & 36.2 & $22.8-39.7$ & CA 2007 \\
\hline $6 \mathrm{AL}$ & QFb.cerz-6AL.1 & barc146-gwm132 & $7.3^{*}$ & 0.39 & 21.4 & 36.2 & $26.3-39.7$ & BW 2007 \\
\hline $6 \mathrm{AL}$ & QFb.cerz-6AL.2 & barc113-wmc553 & $5.5^{*}$ & 0.34 & 17.1 & 65.4 & $57.9-70.8$ & CA 2006 \\
\hline $6 \mathrm{AL}$ & QFb.cerz-6AL.2 & barc113-wmc553 & $8.6^{*}$ & 0.4 & 28.3 & 64.9 & $57.9-68.8$ & BC 2006 \\
\hline $6 \mathrm{AL}$ & QFb.cerz-6AL.2 & barc113-wmc553 & $5.7^{*}$ & 0.32 & 17.9 & 62.4 & $50.7-70.3$ & Mean \\
\hline $6 \mathrm{AL}$ & QFb.cerz-6AL.3 & barc353-gwm169 & $3.4^{*}$ & 0.29 & 12.4 & 90.4 & $78.9-117.2$ & CA 2007 \\
\hline $6 \mathrm{AL}$ & QFb.cerz-6AL.3 & gwm169-BE483091_472 & $3.9^{*}$ & 0.28 & 10.4 & 92.6 & $79.9-125.5$ & BW 2007 \\
\hline 7AS & QFb.cerz-7AS & wmc168-barc 219 & $3.1^{*}$ & -0.29 & 12.6 & 39.1 & $15.5-72.6$ & CA 2006 \\
\hline 7AS & QFb.cerz-7AS & BQ170462_176-barc174 & $4.2^{*}$ & -0.26 & 11.7 & 74.1 & $64.4-84.8$ & BC 2006 \\
\hline $7 \mathrm{AL}$ & QFb.cerz-7AL & $w m c 116-c f d 6$ & $5.8^{*}$ & -0.43 & 22.5 & 165.8 & $151.3-171.1$ & BW 2006 \\
\hline $7 \mathrm{AL}$ & QFb.cerz-7AL & $w m c 116-c f d 6$ & $3.4^{*}$ & -0.26 & 9.8 & 171.1 & $149.8-171.1$ & BC 2007 \\
\hline $7 \mathrm{BS}$ & QFb.cerz-7BS.1 & gwm537-gwm400 & $2.9 \mathrm{~s}$ & 0.22 & 8.5 & 22.3 & $11.4-35.8$ & BC 2006 \\
\hline 7BS & QFb.cerz-7BS.2 & barc72-gwm 297 & $4.4^{*}$ & 0.33 & 12.8 & 59.2 & $53.9-66.8$ & BW 2006 \\
\hline $7 \mathrm{BL}$ & QFb.cerz-7BL.1 & Psy-B1-cfa2257 & $4.3^{*}$ & 0.28 & 12.1 & 198.5 & $184.4-199.3$ & BC 2006 \\
\hline $7 \mathrm{BL}$ & QFb.cerz-7BL.2 & cfa2040-barc1073 & $3.6^{*}$ & 0.25 & 9.5 & 187.6 & 184.4. -199.3 & BC 2006 \\
\hline $7 \mathrm{BL}$ & QFb.cerz-7BL.2 & cfa2040-barc1073 & $4.6^{*}$ & 0.35 & 14.0 & 184.6 & $170.9-190.3$ & BC 2007 \\
\hline
\end{tabular}

a 1, 2, 3, different QTL positions on the same chromosome arm, Fb flour yellow color, cerz Centro de Recursos Naturales de la Zona Semiárida

b Bold closest marker at the peak of the QTL

${ }^{\mathrm{s}} \mathrm{s}$ and $*$ threshold LOD at $p<0.1$ (suggestive) and $p<0.05$ (significant), respectively

${ }^{\mathrm{d}}$ Positive values of additive effect indicate increasing effects of Kofa alleles; negative values indicate increasing effects of UC1113 alleles

e $R^{2}(\%)$ percentage of phenotypic variance explained

f $C A$ Cabildo, $B W$ Barrow, $B C$ Balcarce, Mean pooled data of the six environments

(between the markers dupw4 and barc 170), showing an overlap of the confidence intervals for both traits. The second QTL was located more distally on the long arm, associated with $\mathrm{Fb}^{*}$ (QFb.cerz-4AL.2) in three environments (CA 2006, CA 2007 and BW 2007) and with YPC (QYpc.cerz-4AL.2) in one environment (BW 2006). The 4AL.2 QTL was strongly linked with marker $w m c 219$ for both traits (Tables 2, 3). QTL analysis using the average of all six environments also detected QFb.cerz-4AL.2 (Fig. 1).

Chromosome arms $5 A S$ and $5 A L$

Single QTLs were detected on 5AS and 5AL (Fig. S1), with both positive alleles provided by UC1113. A QTL on 5AS was mapped between wmc350 and 
Table 3 QTL mapping of YPC in a RIL population (UC1113 $\times$ Kofa) grown in six environments in Argentina

\begin{tabular}{|c|c|c|c|c|c|c|c|c|}
\hline $\begin{array}{l}\text { Chromosome } \\
\text { arm }\end{array}$ & $\mathrm{QTL}^{\mathrm{a}}$ & Flanking markers ${ }^{\mathrm{b}}$ & $\mathrm{LOD}^{\mathrm{c}}$ & $\begin{array}{l}\text { Additive } \\
\text { effect }^{\mathrm{d}}\end{array}$ & $\begin{array}{l}R^{2} \\
(\%)^{\mathrm{e}}\end{array}$ & $\begin{array}{l}\text { Peak } \\
\text { position } \\
(\mathrm{cM})\end{array}$ & $\begin{array}{l}\text { 2-LOD } \\
\text { support } \\
\text { interval }\end{array}$ & Environment $^{\mathrm{t}}$ \\
\hline $1 \mathrm{BL}$ & QYpc.cerz-1BL.1 & BE443797_436-barc302 & $3.8 *$ & 0.31 & 10.8 & 52.1 & $38.1-63.0$ & BW 2006 \\
\hline 2AS & QYpc.cerz-2AS & $w m c 382-\mathbf{g w m} 296$ & $2.7 \mathrm{~s}$ & -0.21 & 6.0 & 22.8 & $8.0-47.8$ & BW 2007 \\
\hline $4 \mathrm{AL}$ & QYpc.cerz-4AL.1 & $L p x-A 3-w m c 617$ & $3.9 *$ & -0.31 & 12.0 & 23.3 & $4.8-44.2$ & CA 2006 \\
\hline $4 \mathrm{AL}$ & QYpc.cerz-4AL.2 & wmc219-psr573.2 & $5.4 *$ & 0.34 & 12.0 & 126.2 & $122.6-129.6$ & BW 2006 \\
\hline $5 \mathrm{AS}$ & QYpc.cerz-5AS & wmc350-gwm47 & $3.17 \mathrm{~s}$ & -0.21 & 8.3 & 0.0 & $0.0-17.5$ & BC 2007 \\
\hline $5 \mathrm{AL}$ & QYpc.cerz-5AL & $w m c 727-c f a 2149$ & $3.5^{*}$ & -0.29 & 10.0 & 196.0 & 172.6-205.2 & CA 2006 \\
\hline $5 B L$ & QYpc.cerz-5BL.2 & gwm499-BE495277_339 & $3.8 *$ & -0.26 & 9.0 & 73.3 & $54.5-85.8$ & BW 2007 \\
\hline $6 \mathrm{AL}$ & QYpc.cerz-6AL.1 & barc146-gwm 132 & $7.5^{*}$ & 0.45 & 22.8 & 36.2 & $25.8-38.7$ & CA 2006 \\
\hline $6 \mathrm{AL}$ & QYpc.cerz-6AL.2 & barc113-wmc553 & $7.1 *$ & 0.41 & 16.8 & 66.4 & $58.4-68.8$ & BW 2006 \\
\hline $6 \mathrm{AL}$ & QYpc.cerz-6AL.2 & barc113-wmc553 & $7.2 *$ & 0.41 & 20.9 & 65.9 & $58.4-68.8$ & BC 2006 \\
\hline $6 \mathrm{AL}$ & QYpc.cerz-6AL.2 & barc113-wmc553 & $10.4 *$ & 0.49 & 33.4 & 64.9 & $58.4-68.6$ & CA 2007 \\
\hline $6 \mathrm{AL}$ & QYpc.cerz-6AL.2 & barc113-wmc553 & $13.7 *$ & 0.55 & 42.7 & 64.9 & $59.4-68.1$ & BW 2007 \\
\hline $6 \mathrm{AL}$ & QYpc.cerz-6AL.2 & barc113-wmc553 & $6.5 *$ & 0.3 & 18.6 & 65.4 & $57.4-70.8$ & BC 2007 \\
\hline $6 \mathrm{AL}$ & QYpc.cerz-6AL.2 & barc113-wmc553 & $10.5^{*}$ & 0.43 & 29.9 & 65.4 & $58.4-68.6$ & Mean \\
\hline $6 \mathrm{AL}$ & QYpc.cerz-6AL.3 & barc353-gwm169 & $2.8 \mathrm{~s}$ & 0.29 & 9.8 & 88.4 & $77.6-122.2$ & CA 2006 \\
\hline 7AS & QYpc.cerz-7AS & BQ170462_176-barc174 & $2.9 \mathrm{~s}$ & -0.2 & 6.3 & 74.3 & $55.1-89.3$ & Mean \\
\hline 7AL & QYpc.cerz-7AL & wmc116-cfd6 & $2.9 \mathbf{S}$ & -0.24 & 7.0 & 171.1 & $152.8-171.1$ & BC 2006 \\
\hline 7BS & QYpc.cerz-7BS.2 & barc23-barc72 & $3.6^{*}$ & 0.27 & 9.6 & 58.9 & $43.7-66.8$ & CA 2007 \\
\hline 7BS & QYpc.cerz-7BS.2 & barc23-barc72 & $4.1 *$ & 0.27 & 9.5 & 59.2 & $52.7-62.5$ & BW 2007 \\
\hline $7 \mathrm{BL}$ & QYpc.cerz-7BL.1 & Psy-B1-cfa2257 & $3.4 *$ & 0.3 & 9.3 & 199.0 & $190.5-199.3$ & CA 2006 \\
\hline $7 \mathrm{BL}$ & QYpc.cerz-7BL.1 & Psy-B1-cfa2257 & $2.9 \mathrm{~s}$ & 0.24 & 7.9 & 195.5 & 165.9-199.3 & Mean \\
\hline $7 \mathrm{BL}$ & QYpc.cerz-7BL.2 & cfa2040-barc 1073 & $5.6^{*}$ & 0.29 & 15.0 & 183.9 & 171.4-190.1 & BC 2007 \\
\hline $7 \mathrm{BL}$ & QYpc.cerz-7BL.2 & cfa2040-barc1073 & $3.0 \mathrm{~s}$ & 0.23 & 6.6 & 184.6 & 165.9-199.3 & Mean \\
\hline $7 \mathrm{BL}$ & QYpc.cerz-7BL.2 & $w m c 311-w m c 276$ & $5.4 *$ & 0.42 & 16.9 & 170.4 & $163.0-190.1$ & BW 2006 \\
\hline
\end{tabular}

a 1, 2, 3, different QTL positions on the same chromosome arm, YPC/Ypc yellow pigment content, cerz Centro de Recursos Naturales de la Zona Semiárida

b Bold closest marker at the peak of the QTL

c $\mathrm{s}$ and $*$ threshold LOD at $p<0.1$ (suggestive) and $p<0.05$ (significant), respectively

${ }^{d}$ Positive values of additive effect indicate increasing effects of Kofa alleles; negative values indicate increasing effects of UC1113 alleles

${ }^{\text {e }} R^{2}(\%)$ percentage of phenotypic variance explained

${ }^{\mathrm{f}} C A$ Cabildo, $B W$ Barrow, $B C$ Balcarce, Mean pooled data of the six environments

gwm47 for both traits (QFb.cerz-5AS, QYpc.cerz$5 A S)$. QYpc.cerz-5AL was mapped between wmc727 and $c f a 2149$ in one environment (CA 2006).

\section{Chromosome arm 5BL}

Two QTLs located on chromosome arm 5BL (5BL.1 and 5BL.2) were $32.1 \mathrm{cM}$ apart (Fig. S1). The peak of the 5BL.1 QTL associated with Fb* (QFb.cerz-5BL.1) was close to SSR barc74 in BW 2007, whereas the 5BL.2 peak was located between gwm499 and BE495277_339 and associated with YPC (QYpc.cerz5BL.2) in BW 2007.

\section{Chromosome arm 6AL}

Three QTLs were detected on 6AL (6AL.1, 6AL.2 and 6AL.3) with all positive alleles provided by Kofa 


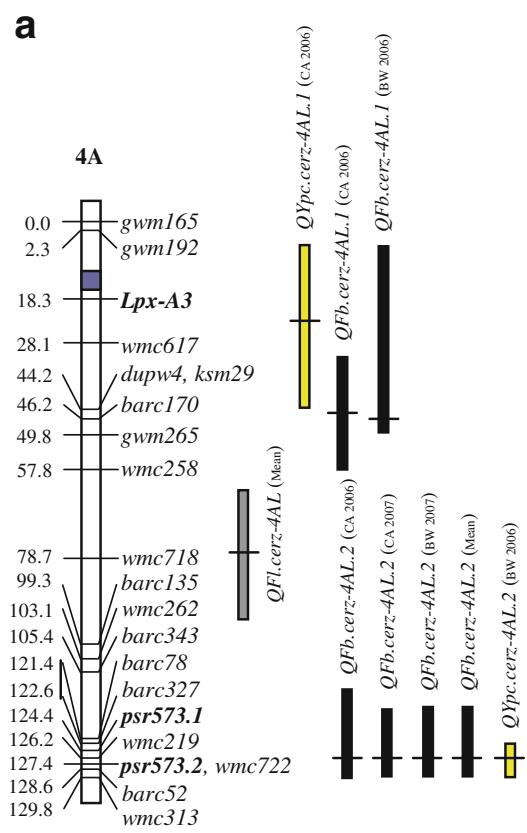

C

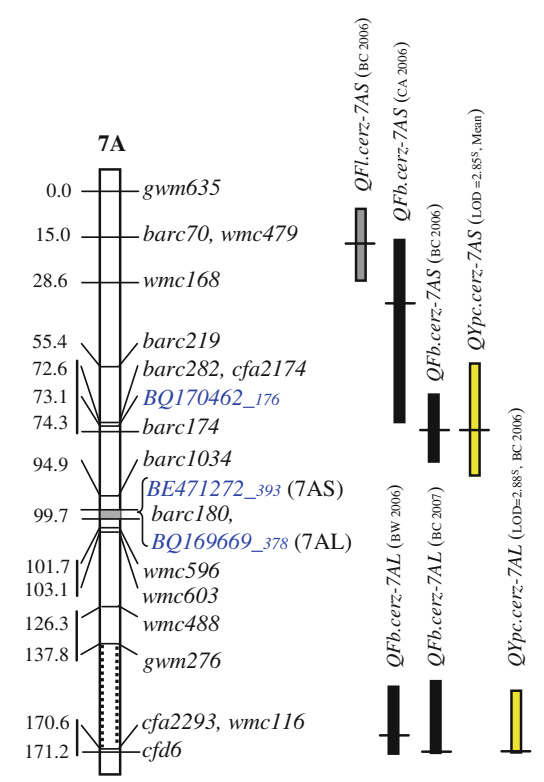

b
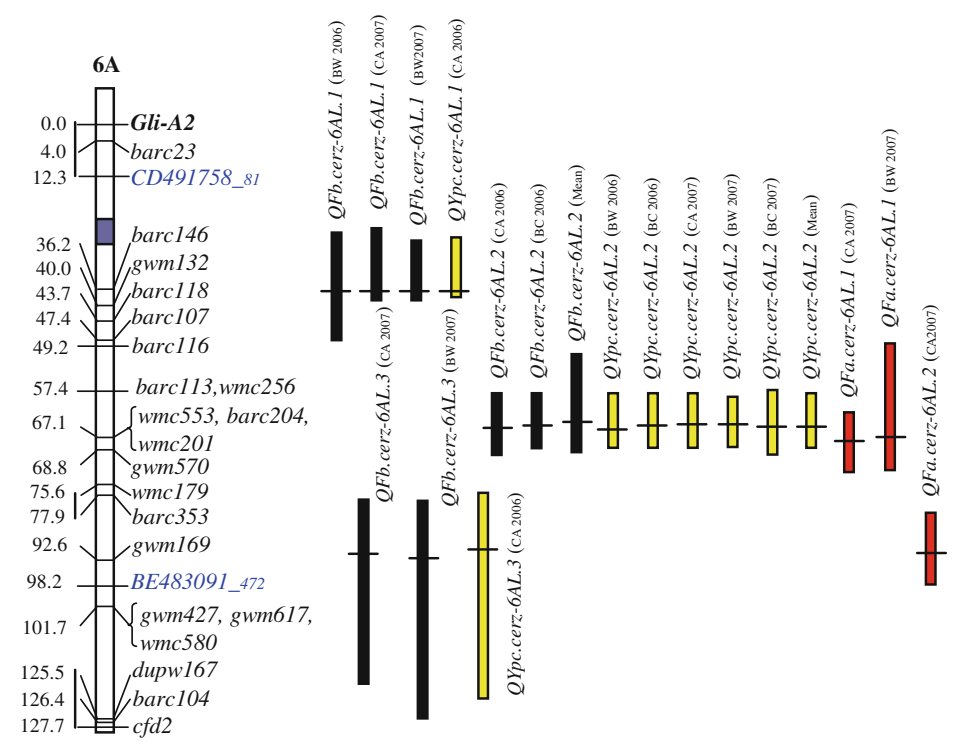

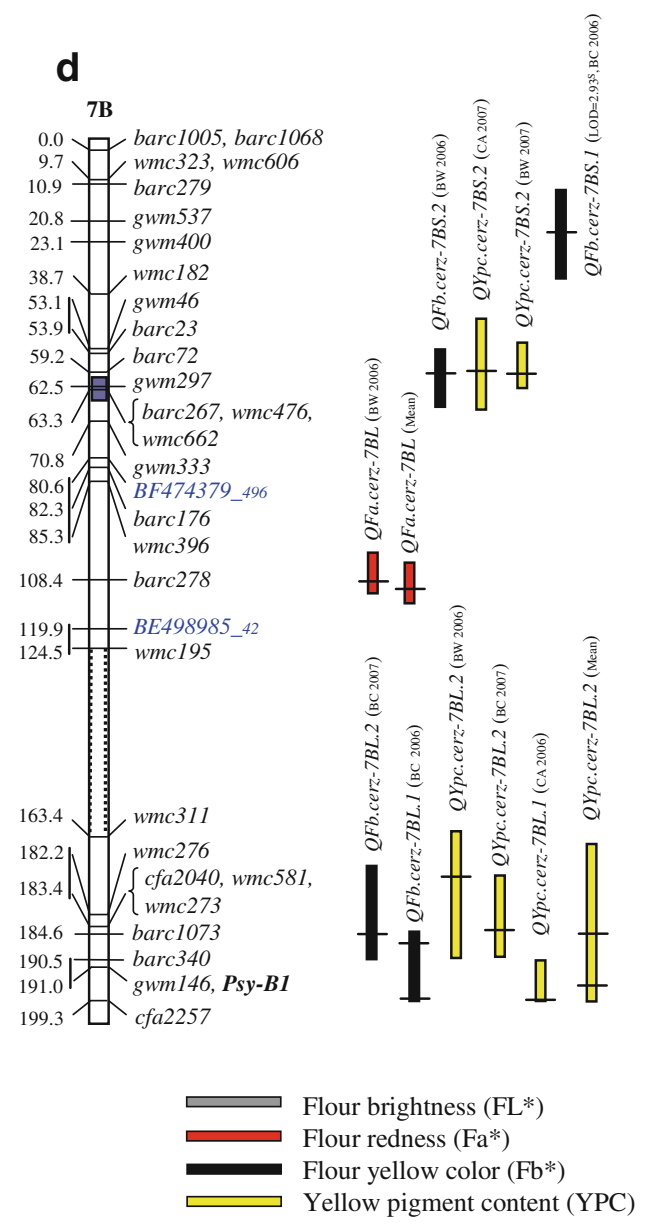


4 Fig. 1 QTLs associated with brightness $(\mathrm{FL} *)$, redness $\left(\mathrm{Fa}^{*}\right)$, yellow color $\left(\mathrm{Fb}^{*}\right)$ and yellow pigment content (YPC) mapped in chromosomes 4A (a), 6A (b), 7A (c) and 7B (d) of the UC1113 $\times$ Kofa RIL population. Centromeres are indicated by grey squares. Markers are on the right and corresponding cumulative genetic distances are indicated on the left. Vertical bars represent QTL confidence intervals with 2-LOD drop offs with the name of the environment at the top. The positions of peaks are indicated by horizontal lines within the QTLs (genetic map from Zhang et al. 2008)

(Fig. 1). These three QTLs individually explained the highest percentages of variation in YPC and $\mathrm{Fb}^{*}$ in the environment in which they were detected. The peak of the 6AL.2 QTL lay between SSRs barc113 and $w m c 553$. This was the most important QTL, displaying a strong and consistent effect on YPC (QYpc.cerz6AL.2) and $\mathrm{Fb}^{*}$ (QFb.cerz-6AL.2) across all six environments, and, in terms of mean values, explaining the highest percentage of the variation in both traits (Tables 2, 3). QFb.cerz-6AL.1, linked to barc 146, was associated with $\mathrm{Fb}^{*}$ in three environments (BW 2006, CA 2007 and BW 2007), whereas QYpc.cerz-6AL.1 showed an effect on YPC only in CA 2006 (Tables 2, 3). Finally, QFb.cerz-6AL.3, linked to gwm169, was significantly associated with $\mathrm{Fb}^{*}$ in two environments (Table 2).

\section{Chromosome arm 7AS}

One QTL was detected on 7AS, with the positive allele provided by UC1113 (Fig. 1). QFb.cerz-7AS between wmc168 and barc219 was significantly associated with $\mathrm{Fb}^{*}$ in CA 2006 (Table 2) and between the markers BQ170462_176 and barc174 in BC 2006. Both peak positions gave overlapping confidence intervals. This QTL was also associated with YPC $($ QYpc.cerz-7AS) as suggestive $(p<0.1)$ from the pooled data, indicating a minor but relatively stable effect on YPC (Tables 2, 3). In addition, this 7AS QTL was detected with LOD values between 2.2 and 2.7 in three environments for YPC and two environments for $\mathrm{Fb}^{*}$.

\section{Chromosome arm 7AL}

A single QTL on 7AL, with the positive allele provided by UC1113, was flanked by wmcl16 and cfd6 (Fig. 1). QFb.cerz-7AL was significantly associated with $\mathrm{Fb}^{*}$ in two environments (BW 2006 and BC
2007), but 7AL was only suggestively associated with YPC (QYpc.cerz-7AL) in BC 2006 (Tables 2, 3).

\section{Chromosome arm $7 B S$}

Two linked QTLs were detected on 7BS, in a region apparently homoeologous to that on 7AS (Fig. 1). Positive alleles for these QTLs were provided by Kofa. QFb.cerz-7BS.1 was associated with $\mathrm{Fb}^{*}$ only as suggestive $(p<0.1)$ in BC 2006, between the markers gwm537 and gwm400. The 7BS.2 QTL was significantly associated with $\mathrm{Fb}^{*}$ and closely linked to barc72 (QFb.cerz-7BS.2) in one environment (BW 2006), and associated with YPC (QYpc.cerz-7BS.2) in two environments. Although not significant, the 7BS.2 peak showed a LOD of 2.65 in the pooled data of $\mathrm{Fb}^{*}$.

\section{Chromosome arm 7BL}

Two closely linked QTLs with positive alleles provided by Kofa were detected in a $35 \mathrm{cM}$ interval on the distal portion of 7BL (Fig. 1). The 7BL.1 peak was flanked by the markers $P s y-B 1$ and $c f a 2257$ and significantly associated with $\mathrm{Fb}^{*}(Q F b$.cerz-7BL.1) in BC 2006 and with YPC (QYpc.cerz-7BL.1) in CA 2006 (Tables 2, 3). The 7BL.2 QTL was located between $c f a 2040$ and barc1073, at a distance of 7.9-15 cM from the 7BL.1 peak. QFb.cerz-7BL. 2 was associated with $\mathrm{Fb}^{*}$ in two environments (BC 2006 and BC 2007) and with YPC (QYpc.cerz-7BL.2) in BC 2007. In BW 2006, the peak of the 7BL.2 QTL was flanked by $w m c 311$ and $w m c 276$ and significantly associated with YPC, with a closely linked second peak between $c f a 2040$ and barc1073. Mapping analysis on the average data detected both QTLs, QYpc.cerz-7BL.1 (LOD = 2.9) and QYpc.cerz-7BL.2 $(\mathrm{LOD}=3.0)(p<0.1)$.

$\mathrm{Fb}^{*}$ and YPC QTLs with epistatic effects or environmental interactions

According to the $F$-statistic, tested on any pair of loci in the genome, a total of 10 digenic epistatic QTLs were detected across nine chromosomes (Table 4). However, no QE or QQE interactions were identified in our analyses. The most important digenic epistatic additive effect was found between the QFb.cerz-1BL.1 and QFb.cerz-1BL.2 QTLs. The new QTL, QYpc.cerz7AS.2, showed QQ interaction for YPC with a QTL on 
Table 4 Epistatic QTLs detected using two-loci QTL analysis

\begin{tabular}{|c|c|c|c|c|c|c|c|}
\hline Trait $^{\mathrm{a}}$ & $\mathrm{QTL}_{\mathrm{i}}^{\mathrm{b}}$ & Flanking markers $s_{i}$ & $\begin{array}{l}\text { Peak } \\
\text { position }_{i}\end{array}$ & $\mathrm{QTL}_{\mathrm{j}}^{\mathrm{b}}$ & Flanking markers ${ }_{j}$ & $\begin{array}{l}\text { Peak } \\
\text { position }_{j}\end{array}$ & $\begin{array}{l}\mathrm{Q}_{\mathrm{i}} \mathrm{Q}_{\mathrm{j}} \\
\text { effect }^{\mathrm{c}}\end{array}$ \\
\hline \multirow[t]{4}{*}{ YPC } & QYpc.cerz-1AL & barc213-cdo393 & 147 & QYpc.cerz-6BL & gwm219-wmc621 & 115.5 & -0.16 \\
\hline & QYpc.cerz-6BL & gwm219-wmc621 & 115.5 & QYpc.cerz-7AS & barc174-barc1034 & 74.3 & -0.12 \\
\hline & QYpc.cerz-2AS & $g w m 249-g w m 71$ & 71.3 & QYpc.cerz-7BS.1 & $g w m 537-g w m 400$ & 22.3 & -0.11 \\
\hline & QYpc.cerz-6AL.2 & barc1165-barc113 & 49.2 & QYpc.cerz-7BS.2 & barc267-gwm333 & 65.3 & -0.12 \\
\hline \multirow[t]{6}{*}{$\mathrm{Fb}^{*}$} & QFb.cerz-4AL.2 & wmc219-psr573.2 & 126.2 & QFb.cerz-6AL.1 & CD491758_81-barc146 & 31.8 & -0.15 \\
\hline & QFb.cerz-4AL.2 & wmc219-psr573.2 & 126.2 & QFb.cerz-7AL & $w m c 116-c f d 6 a$ & 171.1 & 0.10 \\
\hline & QFb.cerz-6AL.1 & CD491758_81-barc146 & 31.8 & QFb.cerz-7AL & $w m c 116-c f d 6 a$ & 171.1 & 0.16 \\
\hline & QFb.cerz-7AL & $w m c 116-c f d 6 a$ & 171.1 & QFb.cerz-7BL.2 & barc1073-barc 340 & 188.1 & 0.13 \\
\hline & QFb.cerz-1BL.1 & BE443797_436-barc302 & 50.6 & QFb.cerz-1BL.2 & GluB1-cfa2129b & 82.6 & -0.20 \\
\hline & QFb.cerz-5BL.2 & BE495277_339-gwm408 & 98.8 & QFb.cerz-7BL & barc176-wmc396 & 82.3 & -0.10 \\
\hline
\end{tabular}

${ }^{\text {a }} \mathrm{Fb} * / \mathrm{Fb}$ flour yellow color, $Y P C / Y p c$ yellow pigment content

b Bold QTL with significant main effect

${ }^{\text {c }} Q_{i} Q_{j}$ effect, epistatic additive effect between $\mathrm{QTL}_{\mathrm{i}} \times \mathrm{QTL}_{\mathrm{j}}$, a negative number indicates decreased trait value; a positive number indicates increased trait value

6BL; the latter was not detected as a main effect QTL for this trait. The main QTL associated with YPC (QYpc.cerz-6AL.2) identified by CIM also showed a QQ effect with another main effect QTL (QYpc.cerz$7 B S .2$ ), whose peak position varied for the epistatic effects from 61.2 to $69.3 \mathrm{cM}$ on 7B. This analysis showed an important QQ effect for $\mathrm{Fb}^{*}$ involving QTLs on chromosomes 7AL (QFb.cerz-7AL) and 7BL (QFb.cerz-7BL.2), and the former QTL also showed epistatic interaction with two additional important genomic regions, viz QFb.cerz-6AL.1 and QFb.cerz4AL.3.

QTL analysis of the FL* color parameter

A genome scan for the $\mathrm{FL}^{*}$ trait using average phenotypic data found four stable QTLs (QFl.cerz2BS, QFl.cerz-3AS, QFl.cerz-3BS and QFl.cerz- $4 A L)$. The QFl.cerz-2BS QTL was identified in three environments (Table 5). However, considering the peak position of this QTL in individual environments, we discarded the possibility of two putative QTLs. This QTL individually explained most of the phenotypic variation in environments CA 2006, BW 2006 and BW 2007, as well as for the mean environmental values (Table 5). QFl.cerz-5BL detected in BC 2007 explained $20.6 \%$ of the variation. The presence of $Q F l$.cerz-7AS was detected in BC 2006.
QTL analysis of the Fa* color parameter

Based on the analysis of the mean values of all six environments, three QTLs associated with $\mathrm{Fa}^{*}$ on chromosomes 1AL, 1BL and 7BL (QFa.cerz-1AL, QFa.cerz-1B.2 and QFa.cerz-7B) explained 12.5, 11.3 and $20.2 \%$ of the phenotypic variation, respectively (Table 5). A QTL was also detected in environment BW 2006 (QFa.cerz-7BL).

With regard to individual environments, QFa.cerz6AL.1 was found in two environments (CA 2007 and BW 2007), and QFa.cerz-6AL.2 was detected in CA 2007. These two QTLs showed pleiotropic effects for both $\mathrm{Fa}^{*}$ and $\mathrm{Fb}^{*}$ (Fig. 1).

\section{Discussion}

ANOVA indicated strong genotypic effects for all traits within each environment, with higher mean square values for YPC and $\mathrm{Fb}^{*}$ than for $\mathrm{Fa}^{*}$ and $\mathrm{FL}^{*}$ (Table S2). Environmental effects and genotype $\times$ environment interactions were detected for all traits (Table S2), indicating that genetic control of flour color in durum wheat is markedly affected by genotype $\times$ environment interaction. However, high broad sense heritability values $(>94 \%)$ for YPC and $\mathrm{Fb}^{*}$ were observed (Table 1), confirming previous 


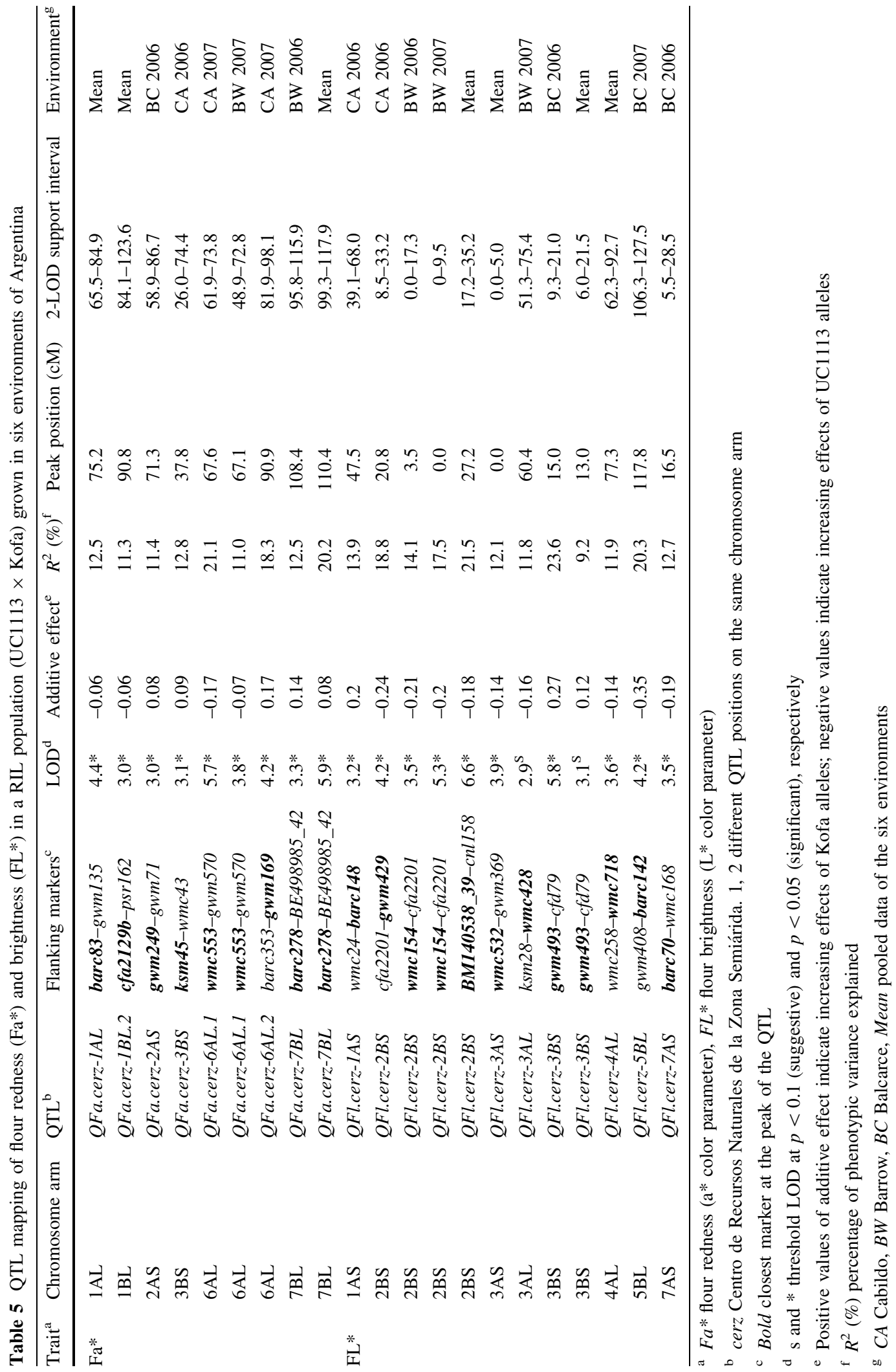


studies showing strong genotypic effects of yellow pigment concentration in durum wheat (Clarke et al. 2006; Digesu et al. 2009; Patil et al. 2008). The high correlation coefficients for YPC and $\mathrm{Fb}^{*}$ across environments suggest that crossover interaction was not important. The population showed transgressive bi-directional segregation for all traits, supported by the presence of genes/QTLs with positive and negative effects from both parents (Table 1). On the other hand, the negative correlation among quality and yield parameters suggests that the environmental effects on grain shape and size could be affecting grain composition and, indirectly, color expression $\left(\mathrm{L}^{*}, \mathrm{a}^{*}, \mathrm{~b}^{*}\right)$ and carotenoid content, as reported by Hessler et al. (2002) (Table S3).

\section{QTLs associated with CIE $b^{*}$ and YPC}

QTL analyses indicated that the correlated $\mathrm{Fb}^{*}$ and YPC traits have complex patterns of inheritance with several genes/QTLs involved. QTLs affecting both traits located on the long arms of group 7 chromosomes were reported in several wheat populations (Pozniak et al. 2007; He et al. 2008; Patil et al. 2008; Howitt et al. 2009), and Psyl genes were proposed as candidate genes for those QTLs (Pozniak et al. 2007). In agreement with previous work using the present mapping population (Zhang and Dubcovsky 2008), the Psy-B1 marker was associated with QYpc.cerz$7 B L .1$ and QFb.cerz-7BL.1 affecting YPC and $\mathrm{Fb}^{*}$, respectively. Although the peak of the 7BL.1 QTL was not coincident with the locus $P s y-B 1$, it was near to this gene in some environments. Our analysis showed the peak of the 7BL.1 QTL was closely linked to cfa2257 located $8.3 \mathrm{cM}$ distal to Psy-B1. However, in the current study, a second QTL (QYpc.cerz-7BL.2; QFb.cerz-7BL.2) was detected between $c f a 2040$ and barc1073 in some environments, instead of the QTL flanked by the Psy-B1 marker. This QTL showed the highest LOD score (3.0) using the mean value of YPC. Based on the information obtained in the Argentinean environments, it was possible to differentiate more clearly these two linked QTLs located in the 7BL region. These results provide additional evidence to support the proposal of Zhang and Dubcovsky (2008) that at least one additional gene affecting flour color traits was present in the 7BL region. Recently, Blanco et al. (2011) reported similar results by detecting two linked QTL, associated with individual carotenoid compounds ( $\beta$-cryptoxanthin and zeaxanthin) on chromosome 7BL. One of them (for zeaxanthin) was associated with the $w m c 311$, the marker located at the peak of QYpc.cerz-7BL.2 in our population in BW 2006. Using wheat deletion lines, Crawford et al. (2008) mapped the (Rab) geranylgeranyl transferase I $\alpha$-subunit gene (RGGT) distally on the long arms of chromosomes 7B and 7D. This gene encoding enzymes involved in the xanthophyll biosynthetic pathway could be a potential candidate for the additional gene proposed by Zhang and Dubcovsky (2008) and in this study.

Kofa carried positive alleles for the QTLs on 7B, whereas UC1113 contributed positive alleles for the 7A QTLs. The QTL mapped on 7AL was reported in different mapping populations of durum and common wheat, explaining a large portion of the yellow pigment variation (He et al. 2008; Patil et al. 2008; Howitt et al. 2009; Blanco et al. 2011). This QTL was also associated with allelic variation of Psy-Al in durum (Singh et al. 2009; Blanco et al. 2011) and common wheat (He et al. 2008; Howitt et al. 2009). In our study, the QFb.cerz-7AL was significantly associated with $\mathrm{Fb}^{*}$ (LOD between 3.8 and 5.4) and weakly associated with YPC, with LOD values between 2.0 and 3.0. Our results showed that the effect of the 7AL genomic region was environmentally unstable, in contrast to the report by Zhang et al. (2008). However, we found that the QFb.cerz-7AL was involved in epistatic interactions with the QTLs QFb.cerz-6AL.1, QFb.cerz-7BL.2 and QFb.cerz-4AL.3. Singh et al. (2009) explored the interaction between $P s y-A I$ and $P$ sy-B1 in durum wheat, without positive results. In the linkage map for the UC1113 $\times$ Kofa population, the Psy-Al locus could not be mapped since it was monomorphic in the cross. Interestingly, the markers flanking the 7AL QTL were coincident with the second QTL reported in this region by Singh et al. (2009). This information suggests that in the epistatic effect QFb.cerz-7AL $\times$ QFb.cerz-7BL.2, a second pair of orthologous genes different from Psyl located on 7L could be involved. Moreover, two linked QTLs affecting both YPC and YC on chromosome 7AL were reported by Blanco et al. (2011). One of these QTLs was flanked by a Psy-Al marker, further supporting the hypothesis of more than one gene on $7 \mathrm{~L}$ associated with these traits.

In addition to the QTLs on 7L, two QTLs on 7S associated with traits $\mathrm{Fb}^{*}$ and YPC were found. This 
also agrees with previous reports. A minor QTL linked to gwm 46 on 7BS was reported by Patil et al. (2008). On our map, this SSR is located $6.1 \mathrm{cM}$ from $\operatorname{barc} 72$ flanking the 7BS.2 QTL and was mapped in the same position as in the consensus linkage map (Somers et al. 2004). Reimer et al. (2008) also identified QTLs on homeologous regions of 7AS and 7BS, flanked by markers linked to our QTLs based on the consensus linkage map (Somers et al. 2004). The QTLs mapped by us in the putative homoeologous region 7AS (QFb.cerz-7AS, QYpc.cerz-7AS) were not reported in previous work using biparental populations. We confirmed the main effect of this QTL on YPC for the overall analysis using QTLNetwork v.2 software (2D genome scan and MCMC options) with an $F$ value of 8.25 (critical value, 4.51). In addition, the 7AS QTL was detected in three and four environments associated with YPC and YC, respectively, using this software. Crawford et al. (2008) reported that genes encoding the enzyme geranylgeranyl transferase II $\beta$-subunit, involved in the carotenoid biosynthetic pathway upstream of the Psy genes, were located in homoeologous regions of $7 \mathrm{~S}$ chromosomes in common wheat. We can consider this group of genes as potential candidates for 7S QTLs. Although the 7S QTLs explained only a minor proportion of the variation, all 7S regions studied (7AS, 7BS.1 and 7BS.2) showed digenic epistatic interactions that contributed to the genetic variance. However, it is possible, because it was detected only as suggestive in only one environment, that the 7BS.1 QTL is not a true YC QTL, but rather the consequence of a pleiotropic effect.

In agreement with Zhang et al. (2008), we found QYpc.cerz-4AL.1 associated with YPC flanking the Lpx-A3 locus. For Fb*, the peak QFb.cerz-4AL.1 was detected closer to barc170, sharing a common confidence interval. This marker was reported by Reimer et al. (2008) as associated with YPC using an association mapping strategy. The second QTL identified in this work, on the distal part of the long arm of the chromosome 4A (4AL.2), was also detected by Zhang et al. (2008) and recently reported in common wheat (Zhang et al. 2009). QFb.cerz-4AL.2 showed a strong effect on $\mathrm{Fb}^{*}$ and was involved in two digenic epistatic interactions, confirming the relevance of this genomic region in flour yellow color determination. In line with this argument, QFb.cerz4AL.2 and QFb.cerz-6AL.2 were the only stable QTLs detected using $\mathrm{Fb}^{*}$ pooled data.
A second Psy gene (Psy2) was assigned to homeologous group 5 chromosomes (Pozniak et al. 2007; Blanco et al. 2009). We detected one QTL on 5AS and two linked QTLs on 5BL (5BL.1 and 5BL.2). $P s y-A 2$ and $P s y-B 2$ were located by Blanco et al. (2009) on the short arms of 5A and 5B by linkage analysis and physical mapping. Psy-A2 shows a similar position to our 5AS QTL. Our results showed that QFb.cerz-5BL.1 was closely linked to the marker barc74, in the same region in which Blanco et al. (2009) positioned the Psy-B2 gene. This finding suggests that the 5BL.1 QTL corresponds with the Psy2 locus. Similar results were obtained by Pozniak et al. (2007). However, Ramya et al. (2010) found that barc74 and gwm371 were associated with a strong QTL involved in kernel width and TKW. Therefore more evidence is required to associate Psy-B2 and the 5BL.1 QTL. In agreement with our results, Reimer et al. (2008) found two linked QTLs on 5B, the second one in a similar position to 5BL.2, based on the common marker gwm408. This marker was also reported flanking a QTL for YPC on 5B in durum wheat (Patil et al. 2008).

Based on our data, and in contrast to previous work (Elouafi et al. 2001; Pozniak et al. 2007; Patil et al. 2008; Zhang et al. 2008; Blanco et al. 2011), QYpc.cerz-6AL.2 and QFb.cerz-6AL.2 were the most relevant QTLs for YPC and $\mathrm{Fb}^{*}$, explaining the highest percentages of phenotypic variation for the mean data for both traits. However, three QTLs were detected on the 6AL chromosome arm. Considering individual environments, QYpc.cerz-6AL.2 was strongly associated with YPC in five of them (Table 3), while the other two QTLs (QFb.cerz6AL.1 and QFb.cerz-6AL.3) jointly explained the highest percentage of variation in $\mathrm{Fb}^{*}$ in two environments (Table 2). The barc146 marker closely linked to the 6AL.1 QTL was reported by Reimer et al. (2008) as significantly associated with YPC. We also found that the interval CD491758_81-barc146 showed an epistatic effect with the 7AL QTL, flanked by the wmc116-cfd6 marker interval. In addition, gwm169 linked to the 6AL.3 QTL was found by Zhang et al. (2006) to flank a QTL on chromosome 6AL that was strongly associated with flour yellowness $\left(b^{*}\right)$ in common wheat. Additionally, a QTL underlying phenotypic variation in grain flour color was also reported on 6BL (Pozniak et al. 2007), but this may be associated with a pleiotropic effect of TKW on grain 
color, as suggested by the authors. Sun et al. (2009) found a TKW QTL in a similar region to the 6AL.3 QTL based on the Somers et al. (2004) consensus map. Interestingly, a molecular study of the carotenoid biosynthetic pathway reported genes encoding the enzyme $\varepsilon$-carotene hydroxylase on homoeologous chromosome arms 6AL, 6BL, 6DL in common wheat (Crawford et al. 2008). This enzyme is involved in the final step of lutein production and it may be a good candidate for the stable 6AL.2 QTL detected on 6AL in the current work. Moreover, further genetic studies should be done in order to determine if the gene encoding the enzyme $\varepsilon$-carotene hydroxylase is responsible for high yellow color, particularly given that the possibility of a QTL associated with grain size (TKW) having a pleiotropic effect on flour color (Pozniak et al. 2007) cannot be discarded.

QTL analyses of the $\mathrm{Fa}^{*}$ and $\mathrm{FL}^{*}$ color parameters

Our results showed a strong QTL associated with FL* located on chromosome arm 2BS (QFl.cerz-2BS). Mares and Campbell (2001) reported a QTL on 2B associated with the flour $\mathrm{L}^{*}$ parameter and initial brightness $(\mathrm{L} * 0 \mathrm{~h})$ of noodle sheets in common wheat. In previous work, QTLs affecting polyphenol oxidase activity (PPO) were reported on homoeologous group 2 chromosomes (Mares and Campbell 2001; Watanabe et al. 2006). It is known that polyphenol oxidases play a major role in the darkening of noodles and other wheat products. Although the main QTLs for PPO were identified on chromosomes $2 \mathrm{~A}$ in durum (Watanabe et al. 2006) and 2D in common wheat (Mares and Campbell 2001) we cannot rule out the possibility that PPO genes affected the FL* parameter in our mapping population. On the other hand, Zhang et al. (2011) located the ZDS gene, encoding the zetacarotene desaturase enzyme associated with yellow pigment content, on chromosome arm 2DL. This enzyme could be causing an indirect effect on FL*. Both possibilities need to be investigated.

Correlation analysis showed a negative relationship between the $\mathrm{Fa}^{*}$ color parameter and the SDS test ( $r=-0.41$, Table S3). To our knowledge, there are no previous reports about this association and it would be valuable to confirm the possibility that the MINOLTA red color $\left(\mathrm{Fa}^{*}\right)$ parameter is a moderate indicator of the SDS test and of gluten strength. Conti et al. (2011) detected four QTLs for SDS test with pleiotropic effects on the $\mathrm{Fa}^{*}$ color parameter in the current work (QFa.cerz-1AL, QFa.cerz-1BL.1, QFa.cerz-3BS and QFa.cerz-6AL.1). These data suggest that gluten composition affects grain color on the $\mathrm{Fa}^{*}$ axis of the color scale. In line with this hypothesis, a QTL for Fa* was associated with the Glu-B3 locus on 1BS that encodes low molecular weight glutenin subunits and affects the SDS test in common wheat (Zhang et al. 2009). The Glu-3 locus was not included in our map; however, QFa.cerz-1BL.1 was linked to the Glu-Bl locus, another storage protein locus associated with gluten strength (Conti et al. 2011), supporting the association between gluten composition and $\mathrm{Fa}^{*}$ observed by Zhang et al. (2009).

\section{Conclusions}

In this work we detected several QTLs affecting flour color traits, particularly for $\mathrm{Fb}^{*}$ and YPC, confirming the complex inheritance of these traits. We validated the main QTLs reported for this population in California by using contrasting environments in a different hemisphere (Argentina), allowing us to find additional QTLs and epistatic interactions involving the most important QTLs. This study allowed a thorough analysis of the environmental stability of QTLs involved in the particular genetic background evaluated.

A novel QTL affecting $\mathrm{Fb}^{*}$ and YPC on chromosome arm 7AS was found. It showed an epistatic effect for YPC with a 6BL QTL of minor effect. It is remarkable that a second putative pair of genes/QTLs affecting $\mathrm{Fb}^{*}$ on $7 \mathrm{AL}$ and $7 \mathrm{BL}$ showed epistatic interaction. Our results differed from previous reports in that the most important QTL affecting $\mathrm{Fb}^{*}$ and YPC was located on chromosome 6AL (6AL.2), with stable performance across environments. This QTL, in addition to QTLs linked to the Psyl genes, could be a useful target for breeding programs aimed at improving durum wheat quality.

Acknowledgments We gratefully acknowledge Dr. Jorge Dubcovsky for providing the molecular map and the mapping population to conduct the present analysis. This project was supported by Consejo Nacional de Investigaciones Científicas y Técnicas (CONICET), Universidad Nacional del Sur (UNS) and Agencia Nacional de Promoción Científica y Tecnológica (ANPCyT, PICT 1011). We thank José Bariffi, Liliana Wehrhahne, Pavan Chand Akkiraju and Patricia Gomez for 
help with field experiments. We also thank Dr. John Rogers from Universidad Nacional del Centro, Bs. As., Argentina for reading the manuscript.

\section{References}

Blanco A, Schiavulli A, Colasuonno P, Gadaleta A, Sonnante G, Pignone D (2009) Mapping of phytoene synthase (psy2) genes on group 5 chromosomes of durum wheat. In: Proceedings of the 19th International Triticeae Mapping Initiative, Clermont-Ferrand, France; August 31-September 4

Blanco A, Colasuonno P, Gadaleta A, Mangini G, Schiavulli A, Simeone R, Digesù A, De Vita P, Mastrangelo AM, Cattivelli L (2011) Quantitative trait loci for yellow pigment concentration and individual carotenoid compounds in durum wheat. J Cereal Sci 54:255-264

Borrelli GM, De Leonardis AM, Platani C, Troccoli A (2008) Distribution along durum wheat kernel of the components involved in semolina colour. J Cereal Sci 48:494-502

Carrera A, Echenique V, Zhang W, Helguera M, Manthey F, Schrager A, Picca A, Cervigni G, Dubcovsky J (2007) A deletion at the $L p x-B 1$ locus is associated with low lipoxygenase activity and improved pasta color in durum wheat (Triticum turgidum ssp. durum). J Cereal Sci 45: $67-77$

Cenci A, Somma S, Chantret N, Dubcovsky J, Blanco A (2004) PCR identification of durum wheat BAC clones containing genes coding for carotenoid biosynthesis enzymes and their chromosome localization. Genome 47:911-917

Churchill GA, Doerge RW (1994) Empirical threshold values for quantitative trait mapping. Genetics 138:963-971

Clarke FR, Clarke JM, Mccaig TN, Knox RE, Depauw RM (2006) Inheritance of yellow pigment concentration in seven durum wheat crosses. Can J Plant Sci 86:133-141

Cong L, Wang C, Li Z, Chen L, Yang G, Wang Y, He G (2009) cDNA cloning and expression analysis of wheat (Triticum aestivum L.) phytoene and $\zeta$-carotene desaturase genes. Mol Biol Reports 37:3351-3361

Conti V, Roncallo PF, Beaufort V, Cervigni GL, Miranda R, Jensen CA, Echenique VC (2011) Mapping of main and epistatic effect QTLs associated to grain protein and gluten strength using a RIL population of durum wheat. J Appl Genet 52:287-298

Crawford AC, Shaw K, Stefanova K, Lambe W, Ryan K, Wilson R, Barclay I, McLean R, Moore C, Francki M (2008) A molecular toolbox for xanthophyll genes in wheat. Proceedings of the 11th International Wheat Genetics Symposium, Australia, 24-29 August. http://ses.library.usyd. edu.au/bitstream/2123/3487/1/P226.pdf

Crawford AC, Stefanova K, Lambe W, McLean R, Wilson R, Barclay I, Francki MG (2011) Functional relationships of phytoene synthase 1 alleles on chromosome 7A controlling flour colour variation in selected Australian wheat genotypes. Theor Appl Genet 123:95-108

Dick JW, Quick JS (1983) A modified screening test for rapid estimation of gluten strength in early-generation durum wheat breeding lines. Cereal Chem 60:315-318
Digesu AM, Platani C, Cattivelli L, Mangini G, Blanco A (2009) Genetic variability in yellow pigment components in cultivated and wild tetraploid wheats. J Cereal Sci 50:10-218

Elouafi I, Nachit MM, Martin LM (2001) Identification of a microsatellite on chromosome 7B showing a strong linkage with yellow pigment in durum wheat (Triticum turgidum L. var. durum). Hereditas 135:255-261

Fares C, Platani C, Tamma G, Leccese F (1991) Microtest per la valutazione del colore in genotipi di frumento duro. Molini d'Italia, Anno XLII, vol 12, pp 19-21

He XY, Zhang YL, He ZH, Wu YP, Xiao YG, Ma CX, Xia XC (2008) Characterization of phytoene synthase 1 gene (Psyl) located on common wheat chromosome 7A and development of a functional marker. Theor Appl Genet 116:213-221

He XY, He ZH, Ma W, Appels R, Xia XC (2009a) Allelic variants of phytoene synthase 1 (Psyl) genes in Chinese and CIMMYT wheat cultivars and development of functional markers for flour colour. Mol Breed 23:553-563

He XY, Wang J, Ammar K, Peña RJ, Xia XC, He ZH (2009b) Allelic variants at the $P s y-A l$ and $P s y-B 1$ loci in durum wheat and their associations with grain yellowness. Crop Sci 49:2058-2064

Hentschel V, Kranl K, Hollmann J, Lindhauer MG, Bohm V, Bitsch R (2002) Spectrophotometric determination of yellow pigment content and evaluation of carotenoids by high-performance liquid chromatography in durum wheat grain. J Agric Food Chem 50:6663-6668

Hessler TG, Thomson MJ, Benscher D, Nachit MM, Sorrells ME (2002) Association of a lipoxygenase locus, $L p x-B 1$, with variation in lipoxygenase activity in durum wheat seeds. Crop Sci 42:1695-1700

Hirschberg J (2001) Carotenoid biosynthesis in flowering plants. Curr Opin Plant Biol 4:210-218

Howitt CA, Cavanagh CR, Bowerman AF, Cazzonelli C, Rampling L, Mimica JL, Pogson BJ (2009) Alternative splicing, activation of cryptic exons and amino acid substitutions in carotenoid biosynthetic genes are associated with lutein accumulation in wheat endosperm. Funct Int Genomics 9:363-376

Kao CH, Zeng Z-B, Teasdale RD (1999) Multiple interval mapping for quantitative trait loci. Genetics 152: 1203-1216

Mares D, Campbell A (2001) Mapping components of flour and noodle colour in Australian wheat. Aust J Agric Res 52:1297-1309

Matsuo RR, Dexter JE (1980) Relationship between some durum wheat physical characteristics and semolina milling properties. Can J Plant Sci 60:49-53

McIntosh RA, Yamazaki Y, Devos KM, Dubcovsky J, Rogers WJ, Appels R (2003) Catalogue of gene symbols for wheat. Proceedings of the 10th International Wheat Genetics Symposium. Instituto Sperimentale per la Cerealicoltura; Rome, vol 4, 77 pp

Patil RM, Oak MD, Tamhankar SA, Sourdille P, Rao VS (2008) Mapping and validation of a major QTL for yellow pigment content on 7AL in durum wheat (Triticum turgidum L. ssp. durum). Mol Breed 21:485-496

Piepho HP (2000) A mixed-model approach to mapping quantitative trait loci in barley on the basis of multiple environment data. Genetics 156:2043-2050 
Pozniak CJ, Knox RE, Clarke FR, Clarke JM (2007) Identification of QTL and association of a phytoene synthase gene with endosperm colour in durum wheat. Theor Appl Genet 114:525-537

Ramya P, Chaubal A, Kulkarni K, Gupta L, Kadoo N, Dhaliwal HS, Chhuneja P, Lagu M, Gupta V (2010) QTL mapping of 1000-kernel weight, kernel length, and kernel width in bread wheat (Triticum aestivum L.). J Appl Genet 51:421-429

Reimer SO, Pozniak CJ, Clarke FR, Clarke JM, Somers DJ, Knox RE, Singh AK (2008) Association mapping of yellow pigment in an elite collection of durum wheat cultivars and breeding lines. Genome 51:1016-1025

SAS Institute Inc (2010) Base SAS ${ }^{\circledR} 9.2$ procedures guide: statistical procedures, 3rd edn. SAS Institute Inc, Cary

Singh A, Reimer S, Pozniak CJ, Clarke FR, Clarke JM, Knox RE, Singh AK (2009) Allelic variation at Psyl-Al and association with yellow pigment in durum wheat grain. Theor Appl Genet 118:1539-1548

Somers DJ, Isaac P, Edwards K (2004) A high-density microsatellite consensus map for bread wheat (Triticum aestivum L.). Theor Appl Genet 109:1105-1114

Sun X-Y, Wu K, Zhao Y, Kong F-M, Han G-Z, Jiang H-M, Huang X-J, Li R-J, Wang H-G, Li S-S (2009) QTL analysis of kernel shape and weight using recombinant inbred lines in wheat. Euphytica 165:615-624

Van Ooijen JW (1992) Accuracy of mapping quantitative trait loci in autogamous species. Theor Appl Genet 84:803-811

Wang DL, Zhu J, Li ZK, Paterson AH (1999) Mapping QTLs with epistatic effects and QTL $\times$ environment interactions by mixed linear model approaches. Theor Appl Genet 99: 1255-1264

Wang S, Basten CJ, Zeng Z (2004) Windows QTL cartographer. V2.0 Program in statistical genetics. North Carolina State
University, North Carolina. http://www.statgen.ncsu.edu/ qtlcart/WQTLCart.htm

Watanabe N, Akond AS, Nachit MM (2006) Genetic mapping of the gene affecting polyphenol oxidase activity in tetraploid durum wheat. J Appl Genet 47:201-205

Yang J, Zhu J, Williams RW (2007) Mapping the genetic architecture of complex traits in experimental populations. Bioinformatics 23:1527-1536

Yi N, Xu S, Allison DB (2003) Bayesian model choice and search strategies for mapping interacting quantitative trait loci. Genetics 165:867-883

Zhang W, Dubcovsky J (2008) Association between allelic variation at the Phytoene synthase 1 gene and yellow pigment content in the wheat grain. Theor Appl Genet 116: 635-645

Zhang LP, Yan J, Xia XC, He ZH, Sutherland M (2006) QTL mapping for kernel yellow pigment content in common wheat. Acta Agron Sin 32:41-45

Zhang W, Chao S, Manthey F, Chicaiza O, Brevis JC, Echenique V, Dubcovsky J (2008) QTL analysis of pasta quality using a composite microsatellite and SNP map of durum wheat. Theor Appl Genet 117:1361-1377

Zhang Y, Wu Y, Xiao Y, He Z, Zhang Y, Yan J, Zhang Y, Xia X, Ma C (2009) QTL mapping for flour and noodle colour components and yellow pigment content in common wheat. Euphytica 165:435-444

Zhang C, Dong C, He X, Zhang L, Xia X, He Z (2011) Allelic variants at the $T a Z d s-D 1$ locus on wheat chromosome $2 \mathrm{DL}$ and their association with yellow pigment content. Crop Sci 51:1580-1590 